\title{
EXAMPLES OF METRIC MEASURE SPACES RELATED TO MODIFIED HARDY-LITTLEWOOD MAXIMAL OPERATORS
}

\author{
Krzysztof Stempak \\ Politechnika Wrocławska, Wydział Matematyki \\ Wyb. Wyspiańskiego 27, 50-370 Wrocław, Poland; Krzysztof.Stempak@pwr.edu.pl
}

\begin{abstract}
We furnish elementary examples of (nondoubling) metric measure spaces, for which the modified Hardy-Littlewood maximal operators $M_{k}$ and $M_{k}^{c}$, uncentered and centered, fail to be weak type $(1,1)$ for $1 \leq k<3$ and $1 \leq k<2$, respectively.
\end{abstract}

\section{Introduction}

The aim of this short note is to complement our previous article [2], where we proved that the modified Hardy-Littlewood maximal operators $M_{3}$ and $M_{2}^{c}$, uncentered and centered, are weak type $(1,1)$; see [2, Theorem 3.1]. This was done in the setting of a general metric measure space $(X, d, \mu)$ with the sole assumption on the Borel measure $\mu$ to be finite on bounded sets (balls of measure zero are admitted). Recall that for a parameter $k \geq 1$ the modified Hardy-Littlewood maximal operator $M_{k}=M_{k, d, \mu}$ is defined by

$$
M_{k} f(x)=\sup _{x \in B} \frac{1}{\mu(k B)} \int_{B}|f| d \mu, \quad x \in X,
$$

where the supremum is taken over all open balls $B$ containing $x$, and the balls of measure zero are omitted. Here $k B$ denotes the ball concentric with $B$ and of radius $k$ times that of $B$. The centered version $M_{k}^{c}=M_{k, d, \mu}^{c}$ is defined analogously but the balls included in the definition of $M_{k}^{c}$ and related to $x \in X$ are centered at $x$ (and of positive measure).

The results of [2, Theorem 3.1], as acknowledged in [2, p. 447], are sharp in the sense that, in general, any $k<3$ or any $k<2$ is not enough in the uncentered or in the centered case, respectively, for $M_{k}$ or $M_{k}^{c}$ to be of weak type $(1,1)$. This was shown by Sawano [1] by a direct construction, see the proof of [1, Proposition 1.1] for the centered case; for the uncentered case it was mentioned in the beginning of [1, Section 3] that a similar construction can be done.

Looking at [1, Section 2.3] one can admit that the construction is tangled. In this note we present extremely elementary examples which prove the abovementioned sharpness.

\section{Examples}

In both examples $X$ is a countable planar graph, connected and acyclic, equipped with the geodesic distance $d$, that is the length of the (unique) minimal path joining

doi:10.5186/aasfm.2016.4119

2010 Mathematics Subject Classification: Primary 42B25.

Key words: Metric measure space, non-doubling measure, modified Hardy-Littlewood maximal operator, weak type $(1,1)$.

Research supported by funds of Faculty of Pure and Applied Mathematics of Technical University of Wrocław, Project \#S50075. 
two points; the distance between two points joined by an edge is, by definition, equal to 1 . The induced topology is discrete and $(X, d)$ is separable. The Borel (nondoubling) measure $\mu$ on $X$ is either the countable measure or a slight modification of it; the measure of any ball is positive and finite.

Example 2.1. (centered case) Consider the graph $X$ with vertices $x_{n}$ and $x_{n j}$, and edges joining $x_{n}$ with $x_{n+1}$, and $x_{n}$ with $x_{n j}$, for any $n \in \mathbf{N}$ and $j \in\{1,2, \ldots, n\}$. See Figure 1. Let $\mu$ be the counting measure on $X, \mu(\{x\})=1$ for $x \in X$. Consider $(X, d, \mu)$ as a metric measure space and fix $1 \leq k<2$. We shall estimate $M_{k}^{c} \delta_{x_{n}}\left(x_{n j}\right)$, where $\delta_{x_{n}}$ denotes the Dirac delta at $x_{n}$. Take $r>1$ such that $k r<2$. Let $B=B\left(x_{n j}, r\right)$. Then $B=\left\{x_{n j}, x_{n}\right\}$ and $k B=B\left(x_{n j}, k r\right)=\left\{x_{n j}, x_{n}\right\}$, hence $\mu(k B)^{-1} \int_{B} \delta_{x_{n}} d \mu=\frac{1}{2}$. Consequently, $M_{k}^{c} \delta_{x_{n}}\left(x_{n j}\right) \geq \frac{1}{2}$ and $\mu\left(\left\{M_{k}^{c} \delta_{x_{n}}>\frac{1}{3}\right\}\right) \geq n$. Since $\left\|\delta_{x_{n}}\right\|_{\ell^{1}(X, \mu)}=1, M_{k}^{c}$ fails to be weak type $(1,1)$.

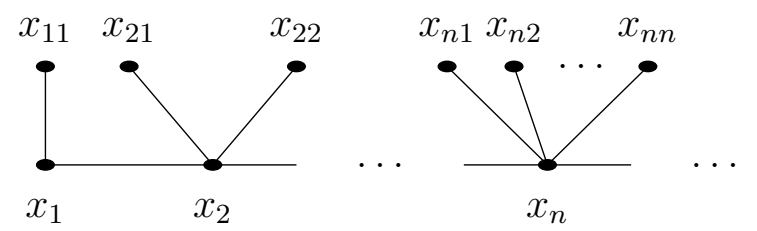

Figure 1.

Example 2.2. (uncentered case) Consider the graph $X$ with vertices $x_{n}, x_{n j}$ and $y_{n j}$, and edges joining $x_{n}$ with $x_{n+1}, x_{n}$ with $x_{n j}$, and $x_{n j}$ with $y_{n j}$, for any $n \in \mathbf{N}$ and $j \in\{1,2, \ldots, n\}$. See Figure 2 (where only the branch starting from $x_{n}$ is shown). Let $\mu$ be the measure on $X$, such that $\mu\left(\left\{x_{n}\right\}\right)=\mu\left(\left\{y_{n j}\right\}\right)=1$, and $\mu\left(\left\{x_{n j}\right\}\right)=\frac{1}{n}$. Consider $(X, d, \mu)$ as a metric measure space, fix $1 \leq k<3$ and choose $1<r<2$ such that $k r<3$. Let $B=B\left(x_{n j}, r\right)$. Then $B=\left\{x_{n}, x_{n j}, y_{n j}\right\}$ and $k B \subset$ $\left\{x_{n-1}, x_{n}, x_{n+1}, x_{n 1}, \ldots, x_{n n}, y_{n j}\right\}$, for $n \geq 2$, say. Therefore $\mu(k B)^{-1} \int_{B} \delta_{x_{n}} d \mu \geq \frac{1}{5}$, and hence $M_{k} \delta_{x_{n}}\left(y_{n j}\right) \geq \frac{1}{5}$. Consequently, $\mu\left(\left\{M_{k} \delta_{x_{n}}>\frac{1}{6}\right\}\right) \geq n$. Since $\left\|\delta_{x_{n}}\right\|_{\ell^{1}(X, \mu)}=$ $1, M_{k}$ fails to be weak type $(1,1)$.

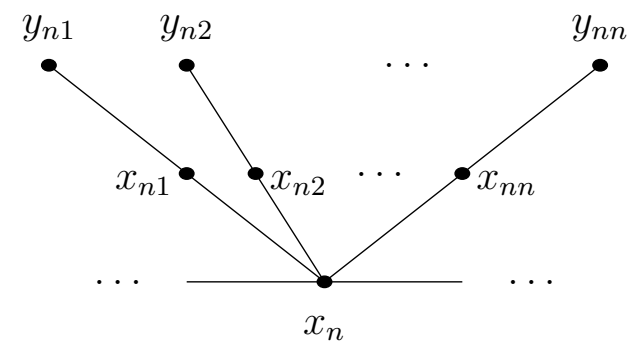

Figure 2 .

Finally, notice that since $\left\|\delta_{x_{n}}\right\|_{\ell^{p}(X, \mu)}=1$ the above argument also shows that $M_{k}^{c}$ for $1 \leq k<2$, and $M_{k}$ for $1 \leq k<3$, fail to be weak type $(p, p)$ for any $1 \leq p<\infty$.

\section{References}

[1] Sawhon, Y.: Sharp estimates of the modified Hardy-Littlewood maximal operator on the nonhomogeneous space via covering lemmas. - Hokkaido Math. J. 34, 2005, 435-458.

[2] Stempak, K.: Modified Hardy-Littlewood maximal operators on nondoubling metric measure spaces. - Ann. Acad. Sci. Fenn. Math. 40, 2015, 443-448. 